\title{
Test and Calibration Beams at the Superconducting Super Collider
}

\author{
Frank Stocker, Howard Fenker, and Ron Schailey \\ Superconducting Super Collider Laboratory* \\ 2550 Beckleymeade Avenue \\ Dallas, Texas 75237
}

\begin{abstract}
Development and operation of the research detectors at the Super Collider will require extensive testing and calibration. This will be done by exposing the detector elements to controlled sourees of particles similar to those which will be encountered when the experiments are taking data. The SSC Lab is designing a test beam facility to meet the needs of the proposed experiments using beam extracted from one or more of the booster accelerators in the SSC complex. In this report we describe the beam requirements and the transport, targeting, and civil systems needed to provide them.
\end{abstract}

\section{INTRODUCTION}

Development and operation of the research detectors at the Super Collider will require extensive testing and on site calibration possibilities over a wide energy range. Todays precision measurments of jets as well as single particles dictates calibration from as low as $1 \mathrm{GeV} / \mathrm{c}^{2}$ up to the highest possible energies. Such a dynamic range can not be covered by a single test beam. The arrangement of the Medium Energy Booster (MEB) and the High Energy Booster (HEB) of the accelerator complex of the SSC provides a possibility to extract two primary beams of 200 $\mathrm{GeV} / \mathrm{c}$ and $2 \mathrm{TeV}$, respectively, and to bring them to a common switchyard as shown in Fig. 1. For financial

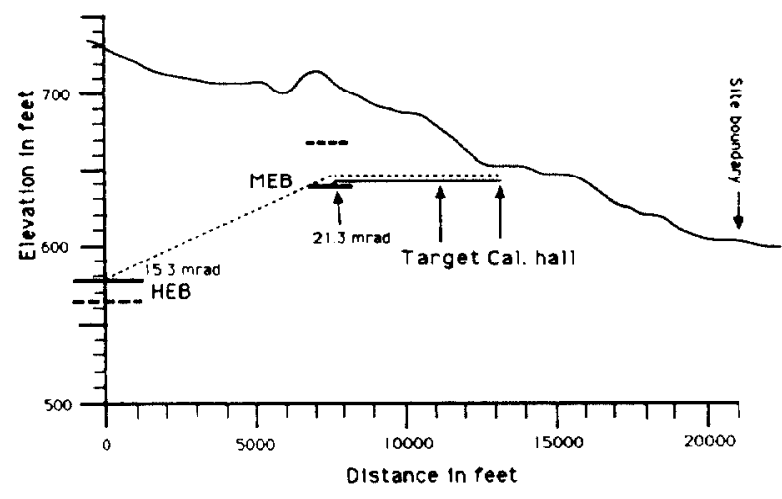

Fig. 1 Elevation View

* Operated by Universities Research Association under contract with the U.S. Department of Energy. reasons the $2 \mathrm{TeV}$ beam will be constructed later since it requires an approximately $8000^{\prime}$ tunnel to bring it to the location of the MEB closer to the surface, not to speak of the many additional benders and quadrupoles. For the same reason we would build only three of the six forseen 200 $\mathrm{GeV}$ beams in a first phase, see Fig. 2. Here we present a study of a possible minimal switchyard for three $200 \mathrm{GeV}$ test beams which has the potential of accomodating a future $2 \mathrm{TeV}$ beam. This layout deviates from earlier scenarios as e.g. described in $[1,2]$. This design however gives us a measure of our flexibility in test beam

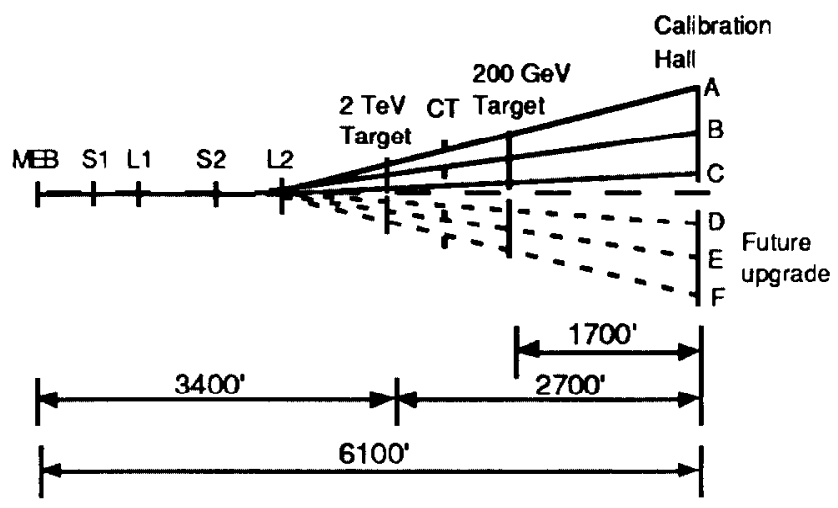

Fig. 2 Test Beam Footprint

geometry. The footprint is so that it may be "duplicated" across the indicated (dashed) symmetry line in Fig. 2 resulting in a total of six $200 \mathrm{GeV}$ beams ond two $2 \mathrm{TeV}$ beams. Dropping one of the planned three $2 \mathrm{TeV}$ beams as in $[1,2]$ reduces the lateral dimensions of the calibration hall and results in major savings of civil construction costs. The $2 \mathrm{TeV}$ beams would lie on top of the middle $200 \mathrm{GeV}$ secondary beams providing the bigger separation of the test stations to cope with the wider muon cone. The presented footprint and layout is only one of several studied scenarios based on the accelerator layout known at that time. The actual layout will emerge as soon as the accelerator elevations and locations will be frozen.

\section{LAYOUT}

Early in the design it became apparent that the main cost driver is real estate. We therefore tried to minimize the required footage of beamline rather than the number of components like benders and septa. The presented 
switchyard is, however, not the shortest possible one for $200 \mathrm{GeV}$, but the shortest one which allows for a $2 \mathrm{TeV}$ upgrade. A detailed analysis of the civil construction requirements however dictated a stretch of the primary beamline in order to put the calibration hall as well as the target hall at locations minimizing the large excavations. This layout also optimizes the part of the switchyard which can be constructed by cut and cover rather than by expensive tunneling.

The muon shielding requires a certain minimal distance of the calibration hall from the target. CASIM [3] simulations indicate $1700^{\prime}$ for the $200 \mathrm{GeV}$ beam and $2700^{\prime}$ for the $2 \mathrm{TeV}$ beam in order to have a maximum muon flux of $150 \mathrm{kHz} / \mathrm{m}^{2}$ as requested by the experimental community. The target hall however represents an important cost driver and we studied a possible scenario with a common target hall (dubbed "CT" in Fig. 2). This represents a compromise between particle yield at low energy and increased muon background for the $2 \mathrm{TeV}$ running.

The above mentioned muon background also drives the distance between the individual calibation bays in the calibration hall. For $200 \mathrm{GeV}$ this distance becomes smaller than what is required by purely mechanical and practical requirements (approx. 30'). The dimensions of the calibration hall have a great influence on the overall costs. We therefore decided to restrict the number of $2 \mathrm{TeV}$ beams to a maximum of two, one at "B" and "E" (see Fig. 2) respectively (The muon cone for $2 \mathrm{TeV}$ is much wider).

Care was taken during the design of the footprint to group as many beam elements as possible in order to minimize the number of magnet enclosures and to shorten cable runs.

Potential fire hazard and serviceability of the power supplies requires them to be located above ground, rather than in the tunnel. To distribute the power, as well as cooling water to the magnets, a number of utility shafts of 5 ' diameter is foreseen along the tunnel.

The split ratio is adjusted by physically moving the septa bank through the beam. The ratio is given by the relative amount of beam that lies to either side of the wires. The septa run at $45 \mathrm{kV} / \mathrm{cm}$, some $90 \%$ of their maximum field, to allow trouble free operation. Care has to be taken in the design of the beam optics so that the beam is wide at the location of the septa. This prevents damaging of the wires, reduces scattering and facilitates the adjustment of the splitting ratio.

The beam transport is done with quadrupole doublets. Twelve quads are needed to transport the 3 beams and take care of constraints like magnet apertures and beam size at the septa. No quads are placed downstream of the splitting station. This would refocus the beam and therefore cancel the (small) separation.

Fig. 3 shows the beam envelope.

\section{SECONDARY BEAMS}

A wide band beam approach has been chosen. Such a beam at Fermilab [4] has an excellent electron yield as well as a high hadron flux.

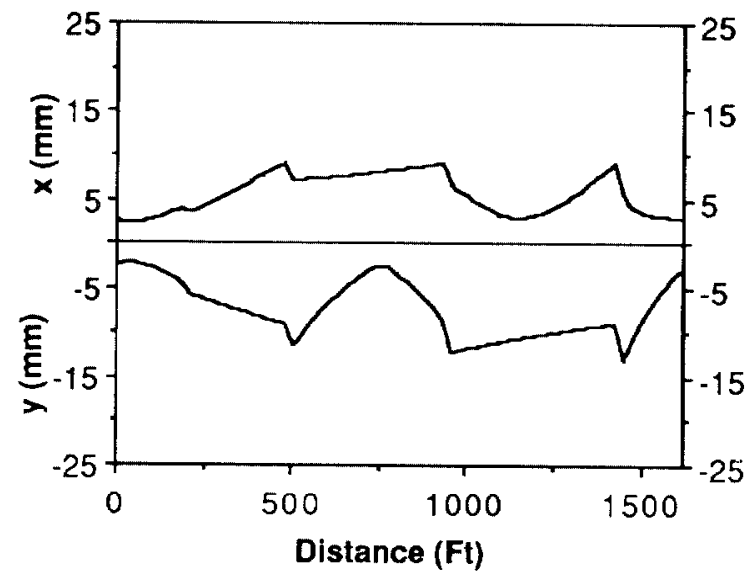

Fig 3 Switchyard Beam Envelope for $200 \mathrm{GeV} / \mathrm{c}$ Beam (3 Sigma)

A triplet system acts as a flux gathering system 150' downstream of the target. It provides a point to parallel imaging. The same enclosure contains the first dipole of the double dogleg system which provides muon shiclding from the production target. A bend angle of $6 \mathrm{mr}$ allows for reasonable real estate and and for good momentum measurement.

Exactly halfway between the target and the calibration hall are the next two magnets of the dogleg structure. The momentum bite and beam intensity may be varied with a variable aperture collimator sitting in the same enclosure.

An enclosure in front of the calibration hall contains the last dog leg magnet and a triplet focusing the beam on the experimental target.

The beam is never momentum dispersed when it passes through a triplet and has, therefore, a large acceptance of about $6 \mu \mathrm{sr} \%$.

All of the secondary vacuum pipe between the enclosures is direct buried stainless steel pipe of $16^{\prime \prime}$ diameter.

\section{YIELDS AND BACKGROUND}

Particle yields were determined using parametrizations of Atherton et al. [6] and taking into account the beamline efficiency by using DECAY TURTLE [5]. Fig. 4 shows expected pion rates for $1 \mathrm{E} 11$ incident protons assuming a 1 
interaction length $\mathrm{Be}$ target. The required rates are easily reached for most of the momenta except for the lowest ones where decay in the secondary beam line introduces a cutoff.Below $3 \mathrm{GeV} / \mathrm{c}$, it is difficult to achieve rates of 100 $\mathrm{Hz}$.

The muon contamination of the pion beam is in the order of 2-3\% over most of the momentum range.

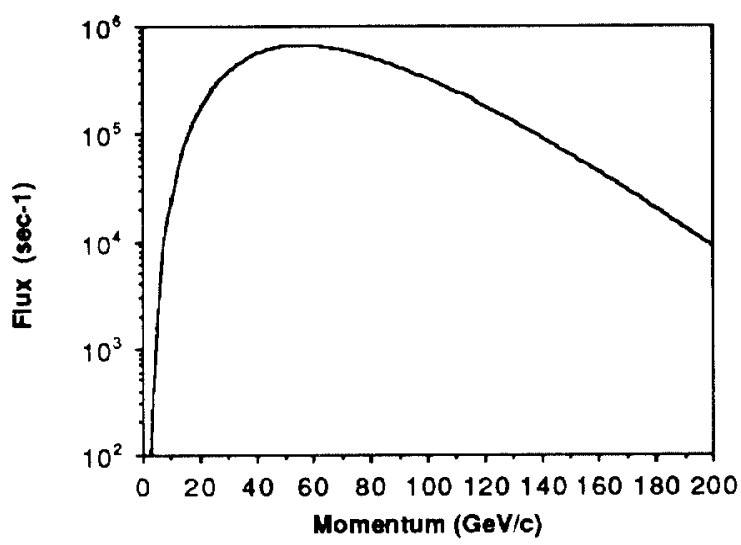

Fig. 4 Pion Rate as a Function of Secondary Momentum, assuming $10^{11}$ Primary Protons $/ \mathrm{sec}$

Electron yields, see Fig. 5, fall sharply for higher momenta. For a primary momentum of $200 \mathrm{GeV} / \mathrm{c}$ the rate drops below $100 \mathrm{~Hz}$ above $150 \mathrm{GeV} / \mathrm{c}$. Should the primary momentum be reduced to $\mathrm{eg} .180 \mathrm{GeV} / \mathrm{c}$ the rate at 150 $\mathrm{GeV}$ would drop to a couple of $\mathrm{Hz}$. This shows the importance of preserving the possibility of ramping the primary beam to $200 \mathrm{GeV} / \mathrm{c}$.

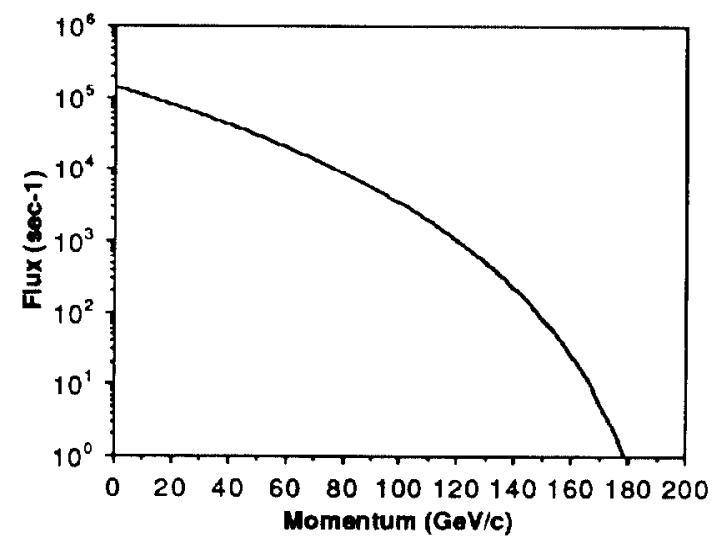

Fig. 5 Electron Rate for $200 \mathrm{GeV} / \mathrm{c}$ Protons assuming $10^{11}$ Primary Protons/sec

The muon background (see Fig. 6) was simulated using CASIM. For the $200 \mathrm{GeV}$ beam the shielding is sufficient to yield approximately $10^{4} \mathrm{~Hz} / \mathrm{m}^{2}$. For the 2 $\mathrm{TeV}$ beam with a common target hall the shortened secondary beamline results in a flux of over $150 \mathrm{kHz} / \mathrm{m}^{2}$. The results shown are for the sweeping plane which explains the dip in the curves.

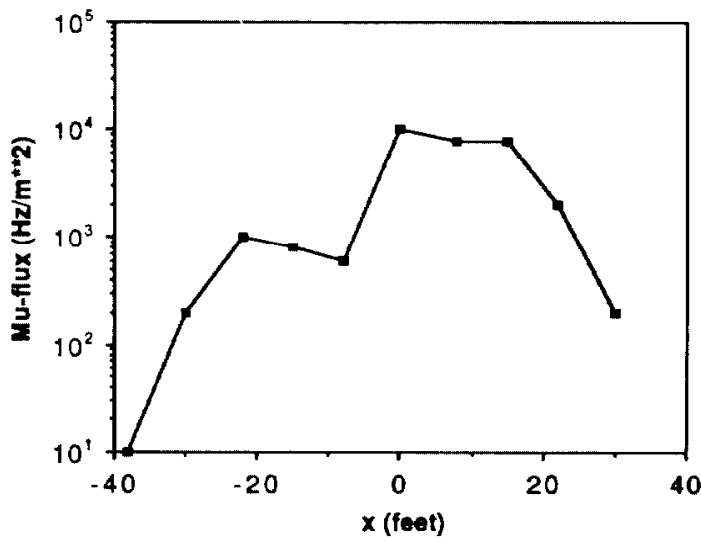

Fig. 6 Muon Flux Density at $1600^{\circ}$ from the Target for $10^{11}$ Protons/sec

We would like to thank R. Stefanski, A. Malensek, J. Bensinger and G. Kalbfleisch for many helpful discussions and $\mathrm{J}$. Bull for providing shielding calculations as well as R. Hoffman for civil construction analysis.

\section{REFERENCES}

[1] A. Autin et al., Calibration Beams at the SSC, SSC Note SSC-230, 1989.

[2] Site-Specific Conceptual Design (SCDR), J. R. Sanford and D. M. Matthews, editors. SSC Report SSCL-SR-1056, 1990.

[3] A. Van Ginneken and M. Awschalom, High Energy Particle Interactions in Large Targets. Fermilab Report FN-272, 1975.

[4] J. Butler et al., Design for a New Wide-Band Neutral Beam for the Tevatron, Fermilab Report TM-963, April 1980.

[5] K. L. Brown et al., DECAY TURTLE, A Computer Program for Simulating Charged Particle Beam Transport Systems, Including Decay Calculations, SLAC Publ. SLAC-246, March 1982.

[6] H. W. Atherton et al., Precise Measurements of Particle Production by $400 \mathrm{GeV} / \mathrm{C}$ Protons on Beryllium Targets, CERN Note CERN 80-07, 1980. 